\section{Metodologia das Pesquisas Populacionais de Saúde Materno-Infantil: uma série transversal realizada no Estado do Ceará de 1987 a 2007}

\section{Method of the Populational Researches in Maternal and Child Health: a transversal series held in the State of Ceará from 1987 to 2007}

Luciano Lima Correia 1

Anamaria Cavalcante e Silva 2

Jocileide Sales Campos 3

Francisca Maria de Oliveira Andrade 4 Dirlene Mafalda Ildefonso da Silveira 5 Márcia Maria Tavares Machado 6 Hermano Alexandre Lima Rocha 7 Antonio José Ledo Alves da Cunha 8

\footnotetext{
1,6,7 Departamento de Saúde Comunitária. Universidade Federal do Ceará. Rua Prof. Costa Mendes, 1609. Fortaleza, CE, Brasil. CEP: 60.430-130. E-mail: hermanoalexandre@gmail.com 2,3,5 Faculdade de Medicina Christus. Fortaleza, CE, Brasil. 4 UNICEF Ceará. Fortaleza, CE, Brasil.

8 Faculdade de Medicina. Universidade Federal do Rio de Janeiro. Rio de Janeiro, RJ, Brasil.
}

\begin{abstract}
Objectives: to describe the methods used in mother-child health studies (PESMIC), over a period of 20 years in Ceará.

Methods: a series of studies carried out over a twenty-year period were examined with regard to the following aspects: a) population-based crosssectional series studies, with a representative sample of 8000 families; b) questionnaires and anthropometric measurements were used, studying the levels and causes of infant mortality, the prevalence of mother and child malnutrition, the duration of breastfeeding, vaccination coverage, the prevalence and management of diarrhea and access to mother-child health services; c) the results obtained were outcome variables (mainly infant mortality) and various determining factors relating to the child and the mother and social and economic conditions.

Results: the response rate was nearly $100 \%$ for the five studies (1986-2007) and there were important changes in socio-demographic and health indicators, an $81 \%$ reduction in the infant mortality rate, a $43 \%$ increase in breastfeeding an immunization rate of $95 \%$. There was also a $243 \%$ increase in overweight In total the five studies covered 177,132 residents, 47,508 women and 13,049 children.

Conclusions: it is recommended that these studies should be continued using the same methodology, making it possible to analyze variations over time.
\end{abstract} Key words Methodology, Epidemiology, Women's health, Child health, Cross-sectional studies
Resumo

Objetivos: descrever os métodos utilizados nas Pesquisas de Saúde Materno-Infantil (PESMIC) no Ceará, durante 20 anos.

Métodos: a série de inquéritos realizados no periodo de 20 anos foi analisada nos seguintes aspectos: a) Estudos transversais em série de base populacional, com amostra representativa de 8000 famílias; b) Utilizou-se questionários e medições antropométricas, estudando-se níveis e causas de mortalidade infantil, prevalência de desnutrição da mãe e da criança, duração da amamentação, cobertura vacinal, prevalência e manejo da diarreia e acesso aos serviços de saúde materno-infantil; c) Obteve-se como resultado as variáveis desfechos (principal mortalidade infantil) e diversos determinantes materno-infantis e socioeconômicos.

Resultados: o índice de resposta foi de quase $100 \%$ nos cinco inquéritos realizados (1986-2007), e ocorreram importantes mudanças nos indicadores sociodemográficos e de saúde; redução de $81 \%$ da taxa de mortalidade infantil, aumento de $43 \%$ da taxa de amamentação; indice de imunização de 95\%. Observou-se aumento de $243 \%$ do indice de sobrepeso. Foram entrevistados no total somado dos cinco estudos: 177.132 residentes, sendo 47.508 mulheres e 13.049 crianças.

Conclusões: recomenda-se a continuidade desses estudos utilizando-se a mesma metodologia, possibilitando analises de variações temporais.

Palavras-chave Metodologia, Epidemiologia, Saúde da mulher, Saúde da criança, Estudos transversais 


\section{Introdução}

No Brasil, como em diversos países do mundo, os inquéritos populacionais são de grande importância para o planejamento, monitoramento e avaliação das ações e políticas de saúde, sendo especialmente úteis para identificar padrões e tendências em saúde quando realizados sequencialmente, gerando séries temporais. ${ }^{1}$ Mesmo com o bom funcionamento dos sistemas de informação em saúde, que geram de modo eficiente dados demográficos, de estatística vitale de procedimentos em saúde, como ocorre atualmente no Brasil, ainda assim os inquéritos populacionais são indispensáveis para se medir a situação de saúde, fatores de risco e comportamentos relacionados à saúde em grupos populacionais específicos. Permitem ainda identificar situações de inequidade em saúde associadas ao acesso, uso e custo dos serviços oferecidos à população. ${ }^{2}$ São bem conhecidos os inquéritos populacionais sobre situação de saúde e comportamentos de risco realizados regularmente pelos EUA, ${ }^{3,4}$ Inglaterra, 5 Alemanha, 6 e praticamente todos os demais países da união européia. ${ }^{7}$ No mundo em desenvolvimento são bem conhecidos os Levantamentos de Saúde e Demografia (DHS), conduzidos pelo governo norteamericano, desde os anos 1980, em 91 países, incluindo o Brasil, com foco na saúde maternoinfantil e planejamento familiar. ${ }^{8}$ No Brasil, os sistemas oficiais de informação em saúde cada vez mais se consolidam, registrando eventos vitais e de atenção em todos os níveis da saúde, sendo complementados pelas PNADS (Pesquisa nacional por amostra de domicílios), que periodicamente incorporam um componente de saúde, ao lado da recente Pesquisa Nacional de Saúde, ora em curso. ${ }^{9}$ Especificamente na Região Nordeste do Brasil, destacam-se a Pesquisa Estadual de Saúde e Nutrição de Pernambuco (PESN), uma série de levantamentos populacionais realizados entre $1991 \mathrm{e}$ 200610,11 e as PESMIC's (Pesquisa Estadual de Saúde Materno-Infantil) realizadas no Estado do Ceará nos anos de 1987, 1990, 1994, 2001 e 2007, ora objeto do presente artigo. ${ }^{12,13}$

Até meados da década de 1980 os indicadores básicos de situação de saúde e nutrição, e de cobertura dos serviços no Ceará eram indisponíveis, defasados ou não confiáveis. Não havia parâmetros, por exemplo, para se estimar taxa de mortalidade infantil do Estado. Assim, em 1986, decidiu-se, por iniciativa governamental e com o apoio do Fundo das Nações Unidas para Infância (UNICEF) e da Universidade Federal de Pelotas (RS), a realização de um diagnóstico de base do estado de saúde da população, denominado PESMIC, direcionado particularmente para o vulnerável grupo maternoinfantil. $14 \mathrm{O}$ estudo populacional visava medir indicadores chave da saúde materno-infantil no Estado, principalmente a taxa de mortalidade infantil e os índices de desnutrição, e, a partir deles, definir as prioridades de ação. Estabeleceu-se ainda o compromisso de que o mesmo estudo seria repetido a cada período de quatro anos, para a avaliação do impacto das intervenções implementadas.

O Estado do Ceará se caracteriza como um dos mais pobres do país, com a inclemência do clima semiárido, que abrange $95 \%$ do seu território, se refletindo marcadamente em sua população de 8,2 milhões de habitantes. Seu desenvolvimento econômico (indústrias e turismo) se concentra basicamente na Região Metropolitana da capital Fortaleza (3,2 milhões de hab.), prevalecendo nas demais regiões a agricultura familiar e os programas governamentais de seguro social, incluindo a complementação de renda. 15 Quanto à saúde, cerca de $85 \%$ da população do estado depende do Sistema Único de Saúde (SUS), que tem conseguido expandir a atenção básica à saúde por meio do Programa Saúde da Família.16-18 Os fenômenos de transição demográfica, epidemiológica e nutricional ocorridos no Estado têm sido adequadamente registradas pela série de 20 anos das PESMIC's, 13 que têm se tornado um instrumento de acompanhamento da situação de saúde e das políticas públicas implementadas no Ceará.

O presente artigo tem por objetivo descrever e avaliar os métodos, procedimentos de coleta dos dados e a participação na série de estudos transversais PESMIC, conduzidas ao longo de 20 anos no Estado do Ceará, visando estimular a realização de outras experiências deste tipo no país.

\section{Métodos}

No presente artigo a metodologia empregada na série de estudos PESMIC é apresentada segundo três elementos principais: a) Desenho do estudo, b) Procedimentos de coleta de dados e c) Resultados da participação no estudo, os quais são detalhados a seguir:

a) Desenho do estudo:

População de estudo;

Plano amostral;

Principais variáveis;

Questionários;

Plano de análise dos dados;

Requerimentos éticos 
b) Procedimentos de coleta de dados:

Logística do trabalho de campo;

Materiais e instrumentos;

Controle de qualidade:

Financiamento dos inquéritos;

c) Resultados da participação no estudo:

Entrevistas realizadas em cada estudo;

Características socioeconômicas da amostra;

A metodologia empregada na série de cinco PESMIC's foi rigorosamente a mesma, mantendo-se inclusive o mesmo tamanho da amostra de 8000 famílias em cada um dos estudos, e os mesmos grupos populacionais. Em todas as pesquisas manteve-se a investigação dos indicadores básicos de natureza socioeconômica e de saúde. Em cada inquérito, entretanto, o enfoque principal do estudo variou, passando da mortalidade infantil, no primeiro estudo (1986), para saúde materna no segundo (1990), saúde reprodutiva no terceiro (1994), saúde da família no quarto (2001) e nutrição maternoinfantil no quinto (2007).

\section{Resultados}

\section{Desenho do estudo}

A série de estudos PESMIC se constituir de levantamentos populacionais do tipo transversal, com abrangência estadual, pesquisando uma amostra representativa de mulheres em idade reprodutiva e de crianças pré-escolares do Ceará

A população dos cinco estudos da série se constituiu das mulheres em idade fértil, na faixa etária de 12 a 49 anos, e das crianças em idade pré-escolar, na faixa de 0 a 35 meses de idade, residentes no Ceará As crianças menores de três anos foram escolhidas como a população infantil alvo na primeira PESMIC (1987) por se constituir num grupo altamente susceptível à morbidade infecciosa à época, para o qual ações de combate à mortalidade infantil deveriam prioritariamente serem dirigidas.

O tamanho da amostra de 8000 domicílios foi inicialmente estabelecido no primeiro estudo de 1987 para a estimativa da taxa de mortalidade infantil no Estado. Este número elevado de domicílios foi necessário para a detecção de um número de óbitos infantis suficiente para o cálculo da taxa. Nos estudos subsequentes, entretanto, o número detectado de óbitos de crianças que haviam morrido nos prévios 12 meses declinou gradativamente, inviabilizando uma estimativa confiável da mortalidade infantil. Mesmo assim o tamanho da amostra de 8000 domicílios foi mantido, uma vez que a queda da taxa de natalidade reduzia cada vez mais o número de crianças encontradas nos domicílios.

Para garantir a representatividade da população estudada, a seleção dos municípios, setores e domicílios foi realizada de forma aleatória, obedecendo a um processo de amostragem de múltiplos estágios. 19 O plano amostral segui as seguintes etapas: a) Amostragem estratificada, para considerar a proporção populacional da capital e do interior do estado; b) Amostragem sistemática, para a seleção dos municípios a serem pesquisados; e c) Amostragem por conglomerados, para a seleção dos domicílios dentro de cada município. Neste último estágio utilizaram-se os setores censitários do Instituto Brasileiro de Geografia e Estatística (IBGE) (áreas geográficas de extensões variáveis, mas com população uniforme de 300 famílias) para a localização dos conglomerados de 20 casas. Esta amostragem foi randomizada; inicialmente estabeleceu-se que 40 municípios seriam sorteados, sendo 13 na Região Metropolitana de Fortaleza, a qual abrange um terço da população do estado, e 27 nas demais regiões do interior do estado, predominantemente rurais. Criou-se uma lista de acordo com o peso populacional, a partir da lista de todos os municípios com suas respectivas populações, sequenciados por região, para garantir uma adequada distribuição geográfica da amostra. Por este processo, um município de grande porte, para ter seu peso populacional respeitado na amostra, poderia ser sorteado mais de uma vez. Desta forma, ao final do processo de amostragem sistemática, 31 municípios compuseram a amostra final, ao invés de 40 , como inicialmente estabelecido.

Em cada município sorteado, 10 setores censitários das zonas urbanas e rurais foram selecionados por sorteio, permitindo assim que todas as áreas, mesmo as mais remotas pudessem ser representadas. Por exemplo, na primeira PESMIC Fortaleza foi selecionada 10 vezes, e, portanto 100 setores censitários do IBGE foram aleatoriamente selecionados nesta cidade.

Uma vez definido o setor, e seu respectivo mapa obtido do IBGE, foi definida por sorteio a localização do conglomerado de 20 casas a serem pesquisadas. Novamente de forma aleatória, foi determinado o ponto de partida do conglomerado, ou seja, o primeiro domićlío a ser visitado. A partir deste, um total de 20 domicílios consecutivos foram visitados.

As questões investigadas sobre as crianças, mulheres e suas famílias, evoluíram conforme novos 
temas de interesse foram entrando na pauta global, nacional ou local da saúde materno-infantil (Tabela 1). As variáveis básicas, entretanto, permaneceram em todos os cinco questionários, possibilitando análises comparativas ao longo da série. As variáveis a seguir se aplicam a essa característica:

Alfabetização materna: capacidade de ler e compreender uma mensagem simples de educação em saúde;

Renda familiar: a renda total mensal ganho por todos os membros da família, expresso em salários mínimos;

Aleitamento materno exclusivo: uso estrito do leite materno, sem água ou chás;

Aleitamento materno predominante: fornecimento de leite materno além de água e /ou chá, sem outros alimentos;

Baixo peso ao nascer: peso de nascimento abaixo de $2500 \mathrm{~g}$;

Baixo peso para idade: peso da criança para a idade abaixo de -2 desvios-padrão;

Atraso de crescimento: altura da criança para a idade abaixo de -2 desvios-padrão;

Emaciação: peso da criança para a estatura abaixo de -2 desvios-padrão;

Diarreia: três ou mais evacuações líquidas nas 24 horas anteriores;

Infecção respiratória aguda baixa: tosse, respiração rápida / difícil e febre, nos últimos 15 dias, segundo o relato da mãe;

Hospitalização por Pneumonia: a criança foi hospitalizada por pneumonia, de acordo com relato da mãe;

Atenção pré-natal: uma ou mais consultas durante a gestação;

Assistência ao parto no hospital: a atenção ao parto, em uma instalação hospitalar.

Os dados tiveram dupla entrada em computador, sendo os dois bancos de dados validados quanto a erros de digitação. No processamento dos dados utilizou-se o software Epi Info 6.04 (CDC/WHO) nas PESMIC's de 1987, 1990 e 1994, e Epi Info 2000 nas de 2001 e 2007. Neste último estudo o programa Anthro foi utilizado para analisar os dados antropométricos, substituindo o programa EpiNut utilizado até então. A análise nutricional gerou os índices de peso por idade, estatura por idade e peso por estatura. Desvios padrões foram calculados para estes índices antropométricos sendo então comparados com os de uma 'população internacional de referência', definida pelo National Center for Health Statistics (NCHS) dos EUA. Na análise dos dados da última PESMIC foi utilizada a referência de crescimento internacional desenvolvida pela Organização Mundial da Saúde (OMS). ${ }^{20}$ Com as medidas antropométricas das crianças foram produzidos os seguintes indicadores de distúrbios nutricionais:

Desnutrição aguda: Peso por Idade $(\mathrm{P} / \mathrm{I})<-2 \mathrm{z}$ scores;

Obesidade: Peso por Idade $(\mathrm{P} / \mathrm{I})>2$ z scores;

Nanismo nutricional: Estatura por Idade $(\mathrm{A} / \mathrm{I})<-$ 2 z scores;

Emaciação: Peso por Estatura $(\mathrm{P} / \mathrm{A})<-2$ z scores.

As medidas antropométricas das mulheres, por sua vez, permitiram o cálculo do Índice de Massa Corporal (IMC), indicador que relaciona peso e altura, de acordo com gênero e idade específicos, segundo parâmetros recomendados pela OMS. Os déficits e excessos encontrados nestes índices antropométricos em relação à população de referência, considerada 'normal', evidenciaram neste estudo os distúrbios nutricionais estudados, com seus respectivos critérios classificatórios. Para a circunferência braquial, o déficit nutricional foi estabelecido como abaixo do percentil 5 , de acordo com o referencial de Frisancho. 21

$\mathrm{Na}$ análise multivariada dos dados as associações entre variáveis dependentes principais, como por exemplo, eventos nutricionais, e variáveis independentes foram exploradas, tendo-se como medida de associação a razão de prevalência. Nesta análise utilizou-se a regressão de Cox, com a estratégia backwards de modelagem.22 A análise de sobrevivência, utilizando-se a técnica de tábuas de vida, foi aplicada às variáveis relacionadas ao aleitamento materno.

Em todas as PESMICs, foram aplicados termos de consentimento livre e esclarecido, submitidos às mulheres e às crianças através de suas mães. Adolescentes menores de 18 anos tiveram consentimento obtido através dos pais ou responsáveis. A partir da quarta PESMIC, de 2001, com a introdução dos Comitês de Ética em Pesquisa através da Resolução $n^{0} 196 / 96$, os protocolos dos estudos foram submetidos a sua apreciação para aprovação.

\section{Procedimentos de coleta de dados}

A equipe técnica do estudo foi constituída de uma Coordenação técnica, institucional e de campo; de um supervisor geral do estudo, de três equipes de pesquisadores de campo, compostas cada uma de um supervisor e oito antropometristas/entrevistadores, todos de nível superior com formação na área de 
Evolução do conteúdo dos questionários das PESMIC's no Estado do Ceará, 1987 - 2007

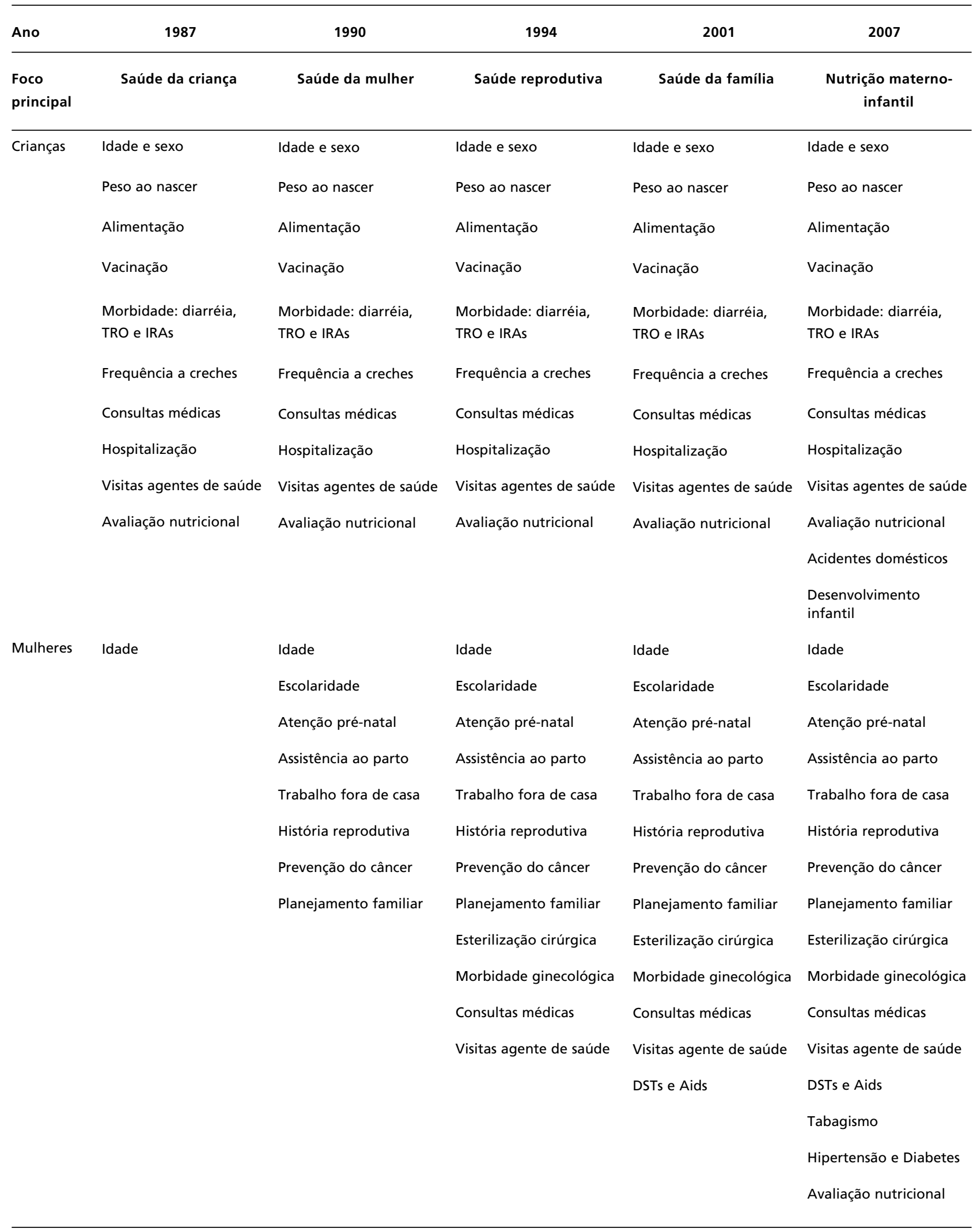


Evolução do conteúdo dos questionários das PESMIC's no Estado do Ceará, 1987 - 2007.

\begin{tabular}{|c|c|c|c|c|c|}
\hline Ano & 1987 & 1990 & 1994 & 2001 & 2007 \\
\hline $\begin{array}{l}\text { Foco } \\
\text { principal }\end{array}$ & Saúde da criança & Saúde da mulher & Saúde reprodutiva & Saúde da família & $\begin{array}{c}\text { Nutrição materno- } \\
\text { infantil }\end{array}$ \\
\hline \multirow[t]{9}{*}{ Família } & Número de pessoas & Número de pessoas & Número de pessoas & Número de pessoas & Número de pessoas \\
\hline & & & & Chefia da família & Chefia da família \\
\hline & & & & $\begin{array}{l}\text { Escolaridade chefe } \\
\text { família }\end{array}$ & $\begin{array}{l}\text { Escolaridade chefe } \\
\text { família }\end{array}$ \\
\hline & Renda familiar & Renda familiar & Renda familiar & Renda familiar & Renda familiar \\
\hline & Água e saneamento & Água e saneamento & Água e saneamento & Água e saneamento & Água e saneamento \\
\hline & & & Acesso ao PSF & Acesso ao PSF & Acesso ao PSF \\
\hline & & & Plano de saúde & Plano de saúde & Plano de saúde \\
\hline & & & & & Segurança alimentar \\
\hline & & & & & Bolsa família \\
\hline
\end{tabular}

PESMIC = Pesquisa Estadual de Saúde Materno-Infantil; TRO= terapia de reidratação oral; IRA= infecção respiratória aguda; DST= doenças sexualmente transmissíveis; Aids= síndrome da imunodeficiência adquirida; PSF= Programa Saúde da Família.

saúde ou nutrição. A equipe de apoio foi constituída por uma secretária, uma auxiliar de pesquisa para revisão dos questionários e por dois digitadores.

Os pesquisadores de campo trabalharam em duplas, cada dupla cobrindo um setor (conglomerado de 20 casas) por dia. O trabalho de campo foi programado para ser executado em 45 dias corridos, ou 34 dias úteis, considerando-se a não ocorrência de imprevistos.

Em cada domicílio, todas as mulheres e crianças nas faixas etárias da pesquisa, nele residentes, tinham suas medidas antropométricas aferidas e os respectivos questionários aplicados.

Após a concluída a coleta de dados no primeiro domicílio, o trabalho de campo prosseguia, seguindo regras específicas: a) O pesquisador visitava as casas obedecendo ao sentido horário; b) Não eram incluídas no conglomerado de 20 casas os estabelecimentos comerciais e as casas sem moradores, sendo estas substituídas por outras vizinhas; c) No caso de famílias ausentes, até três retornos eram realizados na tentativa de obtenção dos dados do domicílio.

As informações foram coletadas utilizando-se três questionários distintos. O primeiro registrou informações de cada domicílio incluído na amostra.
O segundo coletou informações de todas as mulheres de 10 a 49 anos residindo nos domić́lios visitados. Enquanto as mulheres que já haviam engravidado, respondiam ao questionário completo, aquelas ainda sem experiência reprodutiva respondiam somente às questões epidemiológicas básicas. O terceiro questionário, referente à saúde infantil, foi aplicado a todos os responsáveis pelas crianças com menos de três anos de idade residentes nos domicílios visitados.

Para a avaliação do estado nutricional foi realizada a verificação do peso, estatura e circunferência braquial nas mulheres e crianças das faixas etárias pesquisadas. As características dos equipamentos utilizados na antropometria são apresentadas a seguir:

Peso: foi medido por meio das balanças do tipo Salter ${ }^{\circledR}$, com exceção da quinta PESMIC (2007), quando foram substituídas por balanças eletrônicas portáteis tipo Tanita $^{\circledR}$, apropriadas para o uso materno-infantil. A mudança da balança analógica para a eletrônica deveu-se à possibilidade das crianças serem pesadas nos braços da mãe, onde estariam mais calmas, e à facilidade de leitura do peso no visor digital, favorecendo uma redução no 
erro do observador. 20

Estatura: Para a medição do comprimento das crianças foi utilizado um infantômetro na faixa de 30 a $110 \mathrm{~cm}$ de uso portátil e precisão de $1 \mathrm{~mm}$. Crianças menores de 24 meses foram medidas na posição horizontal e as de 24 a 35 meses na posição em pé. A medição da altura das mulheres, por sua vez, foi realizada com um antropômetro portátil na faixa de 65 a $206 \mathrm{~cm}$ e precisão de $0,5 \mathrm{~cm} .{ }^{20}$

Circunferência braquial: foi aferida utilizando-se uma fita métrica especial, tomando-se a medida do braço esquerdo na altura de seu terço médio.

Os equipamentos foram calibrados regularmente utilizando medidas padronizadas, no início do dia e a cada 25 mensurações. 23

A variabilidade inter-antropometristas em procedimentos de aferição foi monitorada através de medições repetidas cegas. Os supervisores acompanharam os antropometristas em suas primeiras sessões de medição.

Durante o trabalho de campo uma sub-amostra de $10 \%$ das crianças e mulheres ( 2 domicílios em cada conglomerado de 20) foram repesadas e tiveram sua estatura remedida pelos supervisores, em uma base cega, como forma de controle de qualidade do trabalho dos antropometristas.

Os questionários foram revisados diariamente pelos supervisores para a identificação de erros de preenchimento e sua correção, caso possível.

\section{Resultados da participação nos estudos}

Os bancos de dados gerados pelas cinco edições da PESMIC dispõem de informações sobre um total de
39.822 famílias, 47.506 mulheres em idade fértil e 13.049 crianças menores de três anos (Tabela 2). Somente um pequeno número de 178 famílias se recusaram a participar da pesquisa, tendo estes casos ocorrido todos nos dois inquéritos mais recentes. Vale salientar que a ausência da família não caracterizou uma recusa, sendo nestes casos o domicílio substituído pelo seguinte.

O número de mulheres disponíveis para as entrevistas nas residências oscilou um pouco de um inquérito para o outro, mantendo-se em torno de 9000, dado que de cada residência foram incluídas todas as mulheres elegíveis (Tabela 3 ). Com relação às crianças, no entanto, o declínio foi acentuado, passando de 4513 crianças menores de três anos encontradas nos domicílios em 1987, para somente 1.533 em 2007, representando uma redução de $66 \%$.

Importantes mudanças ocorreram nos indicadores socioeconômicos e demográficos da população estudada nesse período de 20 anos, conforme pode ser constatado na Tabela 4. Cerca da metade das famílias persistiram recebendo até um salário mínimo mensalmente ao longo do período de 20 anos analisado. Contudo, apesar do indicador econômico não ter melhorado, outros indicadores relevantes para a melhoria da saúde materno-infantil, como o acesso à água potável e a educação feminina, evoluíram de forma expressiva, com aumentos de 136 e de $65 \%$, respectivamente, em duas décadas. A redução do tamanho das famílias foi outro fenômeno constatado neste período, o qual apresenta um forte impacto na economia e na saúde familiar, uma vez que potencialmente representa um pouco mais de recursos e de cuidados para a criança.

Tabela 2

Distribuição dos elementos amostrais das cinco PESMICS's: domicílios, pessoas residentes, mulheres em idade fértil e crianças pré-escolares. Ceará, Brasil, 1987 a 2007.

\begin{tabular}{|c|c|c|c|c|}
\hline Anos & Domicílios & Pessoas residentes & $\begin{array}{l}\text { Mulheres de } \\
10-49 \text { anos }\end{array}$ & Crianças $<3$ anos \\
\hline 1987 & 8000 & 39.653 & 10.743 & 4513 \\
\hline 1990 & 8000 & 31.204 & 8476 & 2861 \\
\hline 1994 & 8000 & 35.935 & 9710 & 2461 \\
\hline 2001 & 7833 & 35.535 & 9754 & 1681 \\
\hline 2007 & 7989 & 34.805 & 8825 & 1533 \\
\hline Total & 39.822 & 177.132 & 47.508 & 13.049 \\
\hline
\end{tabular}

PESMIC = Pesquisa Estadual de Saúde Materno-Infantil. 
Tabela 3

Distribuição das mulheres em idade fértil e crianças pré-escolares nas cinco PESMICS's, de acordo com a faixa etária. Ceará, Brasil, 1987 a 2007

\begin{tabular}{|c|c|c|c|c|c|c|}
\hline Participantes & Faixas etárias & 1987 & 1990 & 1994 & 2001 & 2007 \\
\hline \multirow[t]{5}{*}{ Mulheres (anos) } & 10 a 19 & 3032 & 2225 & 3075 & 3676 & 2072 \\
\hline & 20 a 29 & 3800 & 2838 & 2805 & 2429 & 2519 \\
\hline & 30 a 39 & 2465 & 2066 & 2256 & 2167 & 2312 \\
\hline & 40 a 49 & 1446 & 1347 & 1574 & 1482 & 1922 \\
\hline & Total & 10.743 & 8476 & 9710 & 9754 & 8825 \\
\hline \multirow[t]{4}{*}{ Crianças (meses) } & 0 a 12 & 1401 & 960 & 834 & 605 & 539 \\
\hline & 13 a 24 & 1386 & 997 & 886 & 583 & 532 \\
\hline & 25 a 36 & 1324 & 919 & 741 & 493 & 462 \\
\hline & Total & 4513 & 2861 & 2461 & 1681 & 1533 \\
\hline
\end{tabular}

PESMIC = Pesquisa Estadual de Saúde Materno-Infantil.

Tabela 4

Caracterização socioeconômica e demográficadas famílias participantes das PESMIC's. Ceará, Brasil, 1987 a 2007.

\begin{tabular}{|c|c|c|c|c|c|c|c|}
\hline \multirow[t]{2}{*}{ Indicador } & \multicolumn{5}{|c|}{ Anos } & \multirow[t]{2}{*}{ \% mudança* } & \multirow[t]{2}{*}{$p^{* *}$} \\
\hline & 1987 & 1990 & 1994 & 2001 & 2007 & & \\
\hline $\begin{array}{l}\% \text { de famílias com } \\
\text { renda mensal } \\
\leq 1 \text { salário mínimo }\end{array}$ & 52,5 & 46,5 & 47,3 & 49,4 & 58,1 & $+10,7$ & $<0,001$ \\
\hline $\begin{array}{l}\% \text { de domicílios com } \\
\text { disponibilidade de } \\
\text { água potável }\end{array}$ & 25,30 & - & 25,8 & 67,8 & 59,8 & $+136,4$ & $<0,001$ \\
\hline $\begin{array}{l}\% \text { de mulheres } \\
\text { alfabetizadas }\end{array}$ & 58,40 & 75,10 & 93,0 & 92,7 & 96,4 & $+65,1$ & $<0,001$ \\
\hline $\begin{array}{l}\text { Número médio de } \\
\text { crianças }<3 \text { anos de } \\
\text { idade por mulher }\end{array}$ & 0,74 & 0,55 & 0,56 & 0,32 & 0,25 & $-45,4$ & $<0,001$ \\
\hline
\end{tabular}

PESMIC = Pesquisa Estadual de Saúde Materno-Infantil; *Diferença entre a última e a primeira mensuração; **quiquadrado de tendência. 


\section{Discussão}

Nossos dados mostram ter sido possível realizar com relativo sucesso, através de uma série de estudos transversais de base populacional que atravessou três décadas, o acompanhamento da situação de saúde e nutricional de dois grupos populacionais da maior vulnerabilidade (crianças menores de três anos e mulheres em idade fértil), residentes na região semiárida do Brasil, reconhecidamente a de maior concentração de pobreza do país.

Os bancos de dados dos cinco estudos reunidos proporcionam informações detalhadas sobre $40 \mathrm{mil}$ famílias, que nos permitem análises diversas, inclusive de caráter histórico, sobre a evolução da saúde materno-infantil. A interligação dos três questionários, da mulher, da criança e do domicílio, potencializa mais ainda as possibilidades de análises.

Através das PESMIC's tem sido possível registrar a presença do fenômeno de transição epidemiológica e nutricional napopulação materno-infantil do Ceará, fato este que tem implicações importantes na mudança das políticas públicas de saúde, alimentação e nutrição para a Região Nordeste.24,25 Em duas publicações recentes da PESMIC foram produzidas análises da atual situação nutricional de mulheres e crianças, no que se refere à obesidade e à desnutrição, essenciais para orientar os gestores das áreas sociais e de saúde na formulação de estratégias para o enfrentamento dos dois problemas. 22,26

A experiência das PESMIC'stem gerado, desde o

\section{Referências}

1. Otero UB, Rozenfeld S, Gadelha AJ. Óbitos por desnutrição em idosos, São Paulo e Rio de Janeiro: análise de séries temporais. 1980-1996. Rev Bras Epidemiol. 2001; 4: 191205.

2. Aday LA, Cornelius LJ, Cohen SB. Designing and conducting health surveys: a comprehensive guide. 3 ed. USA: Jossey-Bass Publishers; 2006.

3. Center for Disease Control and Prevention. Department of Health and Human Services. Morbidity and mortality weekly report: surveillance for certain health behaviors among States and selected local areas. Surveillance Summaries. 2013; 62 (1). Disponível em: http://www.cdc.gov/mmwr/pdf/ss/ss6201.pdf

4. Center for Disease Control and Prevention. Department of Health and Human Services. Morbidity and mortality weekly report: youth risk behavior surveillance. Surveillance Summaries. 2014; 63 (4). Disponível em: http://www.cdc.gov/mmwr/pdf/ss/ss6304.pdf?utm_source= rss\&utm medium=rss\&utm campaign=youth-riskbehavior-surveillance-united-states-2013-pdf

5. Craig R, Hirani V. Health Survey for England - 2009: Summary of key findings, health and lifestyles. NHS seu início, um profícuo processo de integração academia-serviços, no qual os resultados das pesquisas têm interagido dinamicamente na formulação de políticas públicas de atenção à saúde maternoinfantil para o Estado, resultando num impacto consistentemente positivo sobre a situação de saúde desta população, reconhecido inclusive internacionalmente. 27

A estratégia de manter-se a mesma metodologia em todos os estudos foi fundamental para garantir uma comparabilidade indiscutível dentro da série temporal. De fato, a cada reedição da PESMIC a sequência inteira de estudos se renova e a série histórica ganha mais importância. Neste sentido a sexta PESMIC está em processo de delineamento, com realização prevista para 2014 , desta vez com foco na assistência neonatal e desenvolvimento infantil.

\section{Agradecimentos}

Ao Ministério da Saúde, ao UNICEF e ao Conselho Nacional de Desenvolvimento Científico e Tecnológico (CNPq) que proporcionaram, em diferentes momentos e formas, o apoio técnico-financeiro indispensável ao desenvolvimento desta série de estudos. Agradecemos igualmente à Secretaria Estadual de Saúde, às prefeituras dos municípios, aos profissionais das áreas acadêmica e assistencial e aos entrevistadores por todo o empenho em viabilizar a realização das pesquisas.
Information Centre for Health and Social Care. UK; 2010

6. Kamtsiuris P, Lange M, Hoffmann R, Rosario AS, Dahm S, Kuhnert R, Kurth BM. The fisrt wave of the German Health Interview and Examination Survey for Adults (DEGS1): sampling design, response, weighting and representativeness. English version Bundesgesundheitsbl. 2013. 56: 62030 .

7. Aromaa A, Koponen P, Tafforeau J, Vermeire C. Evaluation of Health Interview Surveys and Health Examination Surveys in the European Union. Eur J Public Health. 2003; 13 (1): 67-72.

8. BEMFAM, Sociedade Civil Bem-Estar Familiar no Brasil. Pesquisa Nacional Sobre Demografia e Saúde - Brasil, 1996. Programa de Pesquisas de Demografia e Saúde (DHS), Macro International Inc. Rio de Janeiro;1997. 296 p.

9. IBGE (Instituto Brasileiro de Geografia e Estatística). Pesquisa Nacional de Saúde 2013 (PNS). Sistema Integrado de Pesquisas Domiciliares (SIPD/IBGE). Rio de Janeiro; 2012 . 
10. Menezes RCE, Lira PIC, Leal VS, Oliveira JS, Santana SCS, Sequeira LAS, Rissin A, Batista-Filho M Determinantes do déficit estatural em menores de cinco anos no Estado de Pernambuco. Rev Saúde Pública. 2011; 45 (6): 1079-87.

11. Caminha MFC, Batista-Filho M, Serva VB, Arruda IKG, Figueiroa JN, Lira PIC. Tendências temporais e fatores associados à duração do aleitamento materno em Pernambuco. Rev Saúde Pública. 2010; 44 (2): 240-8.

12. Secretaria de Saúde do Estado do Ceará (SESA). Quarta pesquisa de saúde materno-infantil no Ceará, resultados comparativos do período 1987-2001. Fortaleza; 2008.

13. Secretaria de Saúde do Estado do Ceará (SESA). V Pesquisa de Saúde Materno-Infantil do Ceará: 20 anos de acompanhamento, 1987-2007. Fortaleza; 2008.

14. Victora CG, Barros FC, Tomasi E, Ferreira FS, MacAuliffe JF, Silva AC, Andrade FMO, Wilhelm L, Barca DV, Santana S, Gonzales-Richmond A, Shrimpton R. Child health in the States of Ceará, Rio Grande do Norte and Sergipe, Brazil: description of a methodology for the diagnosis of communities. Rev. Saúde Pública. 1991; 25 (3): 218-25.

15. IBGE (Instituto Brasileiro de Geografia e Estatística). Censo Demográfico 2010, Sinopse do Censo e Resultados Preliminares do Universo. Rio de Janeiro; 2011.

16. Victora CG, Barreto ML, do Carmo Leal M, Monteiro CA, Schmidt MI, Paim J, Bastos FI, Almeida C, Bahia L, Travassos C, Reichenheim M, Barros FC, the Lancet Brazil Series Working Group. Health conditions and healthpolicy innovations in Brazil: the way forward. Lancet. 2011; 377 (9782): 2042-53.

17. Macinko J, Starfield B, Erinosho T. The impact of primary healthcare on population health in low- and middle-income countries. J Ambul Care Manage. 2009; 32 (2): 150-71.

18. Macinko J, Marinho de Souza MF, Guanais FC, Silva Simões CC. Going to scale with community-based primary care: an analysis of the family health program and infant mortality in Brazil, 1999-2004. Soc Sci Med. 2007; 65 (10): 2070-80.

19. Barros FC, Victora CG. Epidemiologia da saúde infantil: um manual para diagnósticos comunitários. São Paulo: HUCITEC/UNICEF; 1991. 176 p.
20. De Onis M, Onyango AW, Broeck JVD, Chumlea WC, Martorell R. Measurement and standardization protocols for anthropometry used in the construction of a new international growth reference. Food Nutr Bull. 2004; 25 (Suppl. 1): $\mathrm{S} 27-36$.

21. Frisancho AR. New standards of weight and body composition by frame size and height for assessment of nutritional status of adults and elderly. Am J ClinNutr. 1984; 40 (4): 808-19.

22. Correia LL, Silveira DM, Silva AC, Campos JS, Machado MM, Rocha HA, Cunha AJ, Lindsay AC. Prevalência e determinantes de obesidade e sobrepeso em mulheres em idade reprodutiva na região semi-árida do Brasil. Ciên Saúde Colet. 2011; 16 (1): 133-45

23. Castro V, Moraes SA, Freitas ICM, Mondini L. Variabilidade na aferição de medidas antropométricas: comparação de dois métodos estatísticos para avaliar a calibração de entrevistadores. Rev Bras Epidemiol. 2008; 11 (2): $278-86$

24. Lima ALL, Silva ACF, Konno SC, Conde W, Lisboa BMHA, Monteiro CA. Causas do declínio acelerado da desnutrição infantil no Nordeste do Brasil (1986-19962006). Rev Saúde Pública. 2010; 44 (1): 17-27.

25. Monteiro CA, Benicio MHA, Konno SC, Silva ACF, Lima ALL, Conde WL. Causas do declínio da desnutrição infantil no Brasil, 1996-2007. Rev Saúde Pública. 2009; 43 (1): 35 43

26. Correia LL, Silva AC, Campos JS, Andrade FM, Machado MM, Lindsay AC, Leite AJ, Rocha HA, Cunha AJ. Prevalence and determinants of child under nutrition and stunting in semiarid region of Brazil. Rev Saúde Pública. 2014; 48 (1): 19-28

27. Silva A.C. Evolução da saúde da criança no Ceará: um retrato de dois momentos, 1987 e 1994. Fortaleza: Imprensa Universitária UFC; 1998. 280p.

Recebido em 17 de março de 2014

Versão final apresentada em 21 de agosto de 2014

Aprovado em 30 de setembro de 2014 